\title{
Characterization of TCF3 rearrangements in pediatric B-lymphoblastic leukemia/lymphoma by mate-pair sequencing (MPseq) identifies complex genomic rearrangements and a novel TCF3/TEF gene fusion
}

Ross A. Rowsey ${ }^{1}$, Stephanie A. Smoley ${ }^{1}$, Cynthia M. Williamson ${ }^{1}$, George Vasmatzis², James B. Smadbeck², Yi Ning ${ }^{3}$, Patricia T. Greipp ${ }^{1}$, Nicole L. Hoppman ${ }^{1}$, Linda B. Baughn', Rhett P. Ketterling ${ }^{1}$ and Jess F. Peterson ${ }^{1}$

\begin{abstract}
The TCF3/PBX1 gene fusion is a recurrent genetic abnormality in pediatric B-lymphoblastic leukemia/lymphoma (B-ALL/LBL). While dual-color, dual-fusion fluorescence in situ hybridization (D-FISH) probes can detect TCF3/PBX1 fusions, further characterization of atypical TCF3 FISH patterns as indicated by additional or diminished TCF3 signals is currently limited. Herein we describe the use of a next-generation sequencing assay, mate-pair sequencing (MPseq), to characterize typical and cryptic TCF3/PBX1 fusions and to identify TCF3 translocation partners based on results obtained from our laboratory-developed TCF3/PBX1 D-FISH probe set. MPseq was performed on 21 cases of pediatric B-ALL/LBL with either TCF3/PBX1 fusion, or no TCF3/PBX1 fusion but with additional or diminished TCF3 signals obtained by our PBX1/TCF3 D-FISH probe set. In addition, MPseq was performed on one pediatric B-ALL/LBL case with an apparently normal karyotype and abnormal TCF3 break-apart probe results. Of 22 specimens successfully evaluated by MPseq, 13 cases (59\%) demonstrated TCF3/PBX1 fusion, including three cases with previously undescribed insertional rearrangements. The remaining nine cases (41\%) harbored various TCF3 partners, including six cases with TCF3/ZNF384, and one case each with TCF3/HLF, TCF3/FLI1 and TCF3/TEF. Our results illustrate the power of MPseq to characterize TCF3 rearrangements with increased precision and accuracy over traditional cytogenetic methodologies.
\end{abstract}

\section{Introduction}

Rearrangements involving the TCF3 (previously known as E2A) gene region (19p13.3) are common in both pediatric and adult B-lymphoblastic leukemia/lymphoma (B-ALL/LBL) and account for $\sim 6 \%$ of newly diagnosed

\footnotetext{
Correspondence: Jess F. Peterson (peterson.jess@mayo.edu)

${ }^{1}$ Division of Laboratory Genetics and Genomics, Department of Laboratory

Medicine and Pathology, Mayo Clinic, Rochester, MN, USA

${ }^{2}$ Center for Individualized Medicine-Biomarker Discovery, Mayo Clinic, Mayo

Clinic, Rochester, MN, USA

Full list of author information is available at the end of the article.

These authors contributed equally: Rhett P. Ketterling, Jess F. Peterson
}

cases $^{1-4}$. The $P B X 1$ gene $(1 \mathrm{q} 23)$ is the most common translocation partner for TCF3, resulting in TCF3/PBX1 gene fusion, and is currently classified in the $\mathrm{WHO}$ as a recurrent genetic abnormality in $\mathrm{B}-\mathrm{ALL} / \mathrm{LBL}^{4}$. Fusion of $T C F 3 / P B X 1$ is usually generated from a reciprocal $t(1 ; 19)$ (q23;p13.3) and results in a $5^{\prime} T C F 3 / 3^{\prime} P B X 1$ fusion gene located on the $\operatorname{der}(19) \mathrm{t}(1 ; 19)$ chromosome $\mathrm{e}^{4-6}$. Interestingly, the majority of $1 ; 19$ translocations $(\sim 80 \%)$ only present with the $\operatorname{der}(19) t(1 ; 19)$ as observed by conventional chromosome and/or fluorescence in situ hybridization (FISH) studies $^{5}$. Several rare TCF3 gene fusion 
partners have been described, most commonly ZNF384 (12p13) and $H L F(17 q 21)^{7-9}$. Since highly variable prognoses are associated with the various TCF3 translocation partners (TCF3/HLF fusion has an extremely poor prognosis in contrast to the favorable/standard risk for TCF3/ $P B X 1$ fusion), the characterization of TCF3 partners is essential ${ }^{4}$.

To detect the recurrent TCF3/PBX1 gene fusion in B-ALL/LBL, our laboratory developed and validated a dual-color, dual-fusion FISH (D-FISH) TCF3/PBX1 probe set for clinical application ${ }^{10}$. To further characterize $T C F 3 / P B X 1$ fusions associated with discordant chromosome results and the TCF3 rearrangements with additional or diminished TCF3 signals obtained by our TCF3/ $P B X 1$ D-FISH probe set, we utilized a next-generation sequencing (NGS) strategy, mate-pair sequencing (MPseq). This novel NGS-based technology enables the characterization of chromosomal rearrangements with significantly higher resolution and precision compared to conventional cytogenetic methodologies, including chromosome and FISH studies. Herein, we report conventional chromosome, FISH and the molecular characterization of each TCF3 rearrangement by MPseq from 22 patients with pediatric B-ALL/LBL. This study provides a molecular window into the complexity resulting in TCF3/PBX1 fusion and also highlights the importance of characterizing variant TCF3 partner genes by NGS methods such as MPseq.

\section{Materials and methods Patient selection}

Following institutional review board approval, a retrospective review of the Mayo Clinic cytogenetic database was performed to identify bone marrow samples with abnormal results evaluated by our TCF3/PBX1 D-FISH probe set. Ten pediatric patient cases were chosen that had typical concordant abnormal chromosome and abnormal TCF3/PBX1 fusion D-FISH signal patterns, including six patients with a balanced $t(1 ; 19)$ and a 1R1G2F D-FISH signal pattern (Table 1; patients 1-6), and four patients with an unbalanced $\operatorname{der}(19) \mathrm{t}(1 ; 19)$ and a 2R1G1F D-FISH signal pattern (Table 1; patients 7-10). In addition, three pediatric patient cases were included with discordant abnormal chromosome results versus DFISH results (Table 1; patients 11-13). Patients with atypical D-FISH signal patterns including additional or diminished TCF3 signals in the absence of TCF3/PBX1 fusion were also identified, and represent a total of eight cases (Table 1; patients 14-21). One case with an apparently normal female karyotype and TCF3 rearrangement obtained via a TCF3 break-apart probe (BAP) strategy was also included in our study (Table 1; patient 22). In total, 22 cases of pediatric B-ALL/LBL with TCF3 abnormalities detected by FISH were included in our study, and evaluated by MPseq on either fresh or fixed cell pellets from bone marrow aspirate specimens.

\section{Conventional chromosome analysis}

Bone marrow aspirate specimens were cultured in unstimulated media, harvested and banded utilizing standard cytogenetic techniques according to specimenspecific protocols ${ }^{11}$. Twenty metaphases were analyzed when available.

\section{Fluorescence in situ hybridization}

FISH analysis was performed on bone marrow aspirates specimens following standard FISH pretreatment, hybridization and fluorescence microscopy protocols utilizing a laboratory-developed $T C F 3 / P B X 1$ D-FISH probe set. Details regarding probe development and performance have been previously described ${ }^{10}$.

\section{Mate-pair sequencing}

MPseq was performed using the Illumina Nextera Mate-Pair library protocol (Illumina, San Diego, CA) and sequenced on the Illumina HiSeq 2500. Sequence data were aligned to hg38 using BIMA, and a custom bioinformatics tool was used to filter the alignments and identify junctions. Detailed methods can be found in Drucker et al., Johnson et al., and Smadbeck et al. ${ }^{12-14}$.

\section{Results}

Conventional chromosome, FISH, and MPseq results from all 22 patients (age range: 1-16 years) are presented in Table 1 . For the 13 patients with TCF3/PBX1 fusion, six cases had a balanced $t(1 ; 19)$ by chromosomes and a double fusion by D-FISH (patients 1-6), four cases had an unbalanced $\operatorname{der}(19) \mathrm{t}(1 ; 19)$ by chromosomes and a single fusion by D-FISH (patients 7-10), and three cases had seemingly discrepant chromosome and FISH results, indicated by an unbalanced $\operatorname{der}(19) \mathrm{t}(1 ; 19)$ by chromosomes versus a double fusion signal pattern by D-FISH (patients 11-13).

MPseq confirmed the balanced (patients 1-6) and unbalanced (patients 7-10) 1;19 translocations observed by both chromosome and FISH studies. In addition, MPseq revealed insertional rearrangements accounting for the discrepant chromosome and FISH results in the three cases with $\operatorname{der}(19) t(1 ; 19)$ and two fusion signals (patients 11-13) (Fig. 1a-e). The MPseq results explained serial metaphase FISH, which had documented TCF3/PBX1 fusion located on a "normal" copy of chromosome 1q in each case, suggesting a cryptic insertional rearrangement.

The nine remaining samples (patients 14-22) had additional or diminished TCF3 signals in the absence of $T C F 3 / P B X 1$ fusions, and only four of seven cases (patients $14,17,18$ and 21) with available chromosome studies had abnormalities involving the 19p13 chromosomal region. 


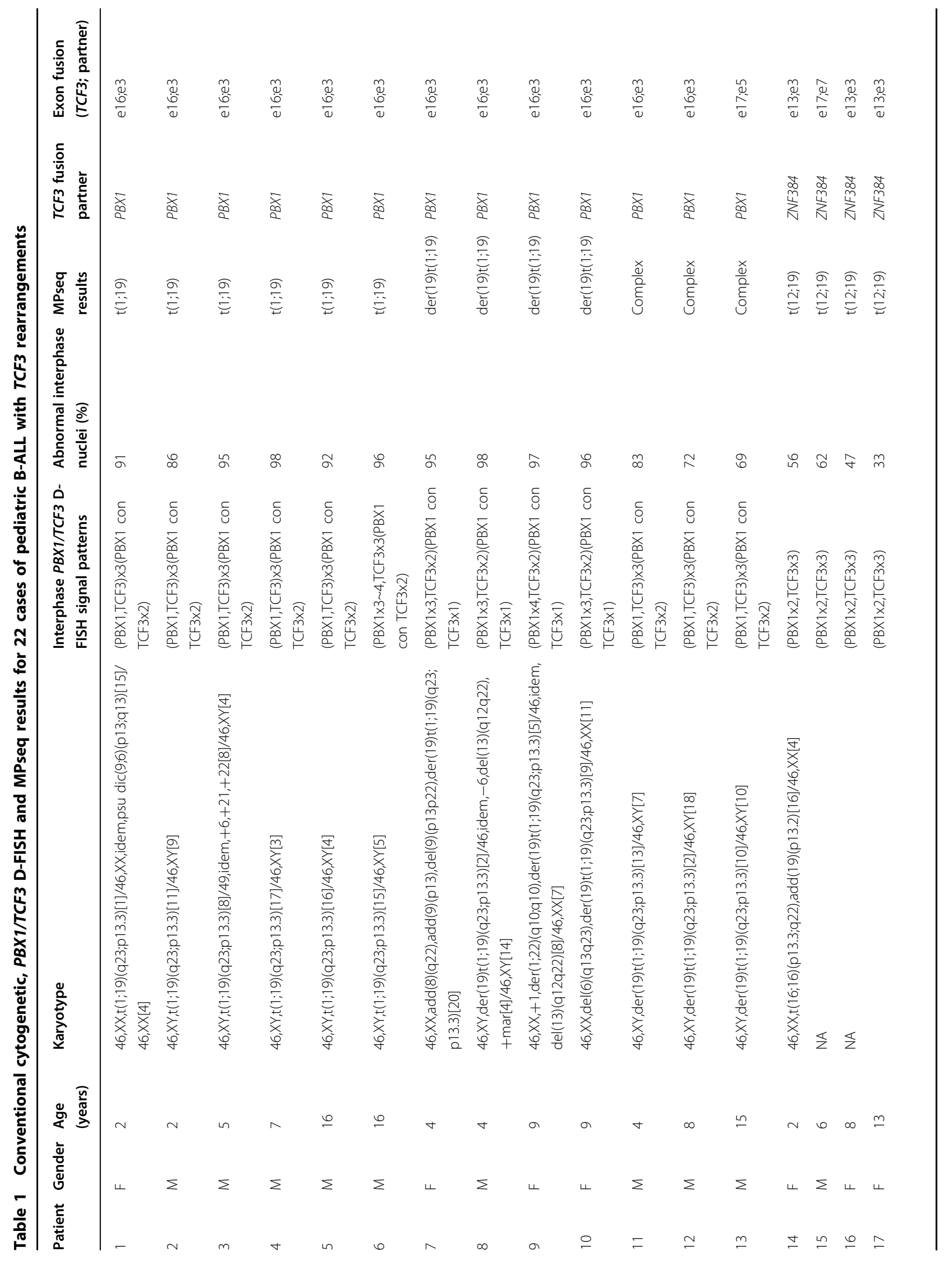




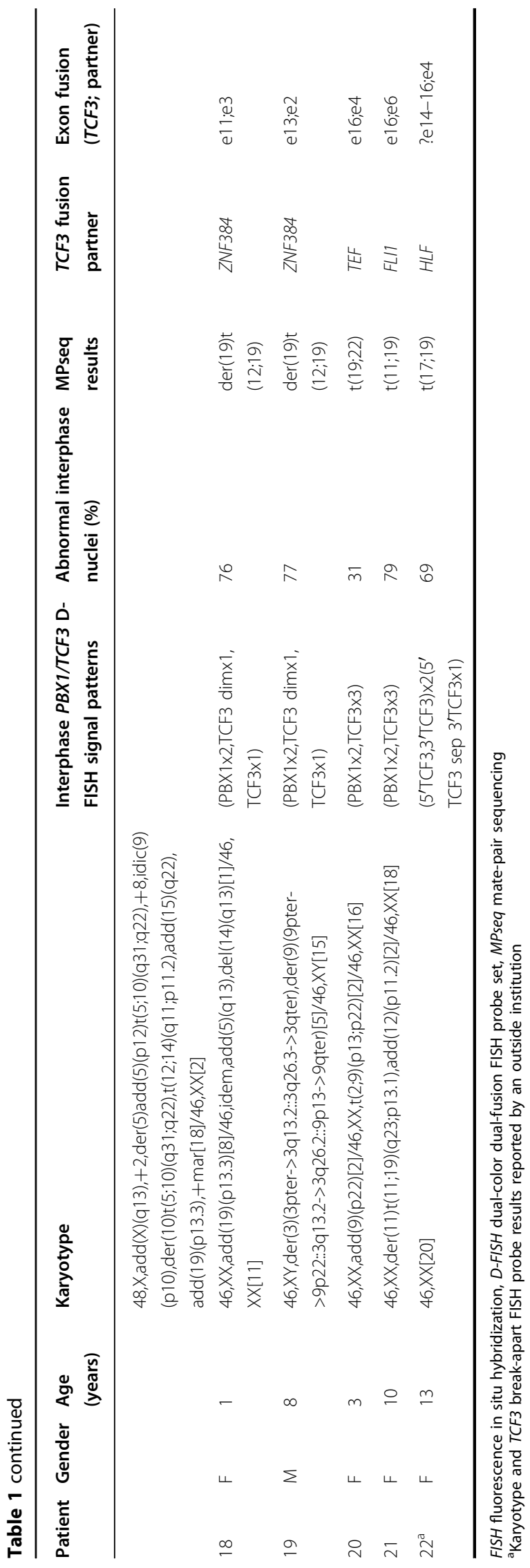

Among these nine cases, MPseq detected variant TCF3 gene partners, including six cases with ZNF384 (patients 14-19) (Fig. 2a-d), and one case each with the following three genes: TEF (patient 20) (Fig. 3a-c), FLI1 (patient 21) and HLF (patient 22). Moreover, MPseq indicated all rearrangements were predicted to create in-frame gene-gene fusions, including all three potential TCF3 exons (14-16) described in patient 22.

\section{Discussion}

Herein, we demonstrate the ability for MPseq to molecularly characterize $22 \mathrm{~B}-\mathrm{ALL} / \mathrm{LBL}$ specimens that were either positive for $T C F 3 / P B X 1$ fusion or displayed an atypical TCF3 FISH pattern with additional or diminished TCF3 signals obtained by our TCF3/PBX1 $D$-FISH probe set. Precise identification of these rearrangements is critical as there are significant prognostic differences between $T C F 3 / P B X 1$ fusion and the less common TCF3/HLF fusion, the latter having a significantly unfavorable prognosis ${ }^{4,9,15}$.

To our knowledge, this represents only the second report of TCF3/FLI1 fusion in the literature and the first to report TCF3/TEF fusion ${ }^{16}$. TCF3 is a helix-loop-helix (HLH) transcription factor critical for lymphopoiesis in B- and T-cell lineage development $^{17,18}$. When the transactivation domain of TCF3 (N-terminal region) is separated from its intrinsic DNA-binding domain (C-terminal) and forms a chimeric fusion with a DNA-binding domain from a new gene partner, the subsequent chimeric fusion protein acts as an oncogenic driver ${ }^{17,18}$. Functional similarities have been described between the DNA binding domains of the $T E F$ and $H L F$ genes, the latter being the TCF3 gene fusion partner observed in the prognostically unfavorable $t(17 ; 19)^{19,20}$. Since these two TCF3 translocation partners share distinct functional similarities, this suggests that the $\mathrm{t}(19 ; 22)$ likely represents the oncogenic fusion and similarly implicates TEF as another potentially aggressive partner of TCF3, although additional follow-up for our patient and identification of the 19;22 translocation and outcomes in other patients are necessary.

Ten pediatric B-ALL/LBL cases with both balanced (patients 1-6) and unbalanced (patients 7-10) TCF3/ $P B X 1$ fusions, as initially identified by conventional chromosome and TCF3/PBX1 D-FISH studies, were readily confirmed by MPseq. In addition, three TCF3/ PBX1 fusion cases (patients 11, 12 and 13) with discrepant chromosome $[\operatorname{der}(19) \mathrm{t}(1 ; 19)]$ and D-FISH results (two fusions) were further characterized by MPseq beyond the capability of traditional chromosome and FISH methodologies. In all three cases, DFISH studies suggested a typical 1;19 translocation (two fusion signal pattern); however, the conventional 


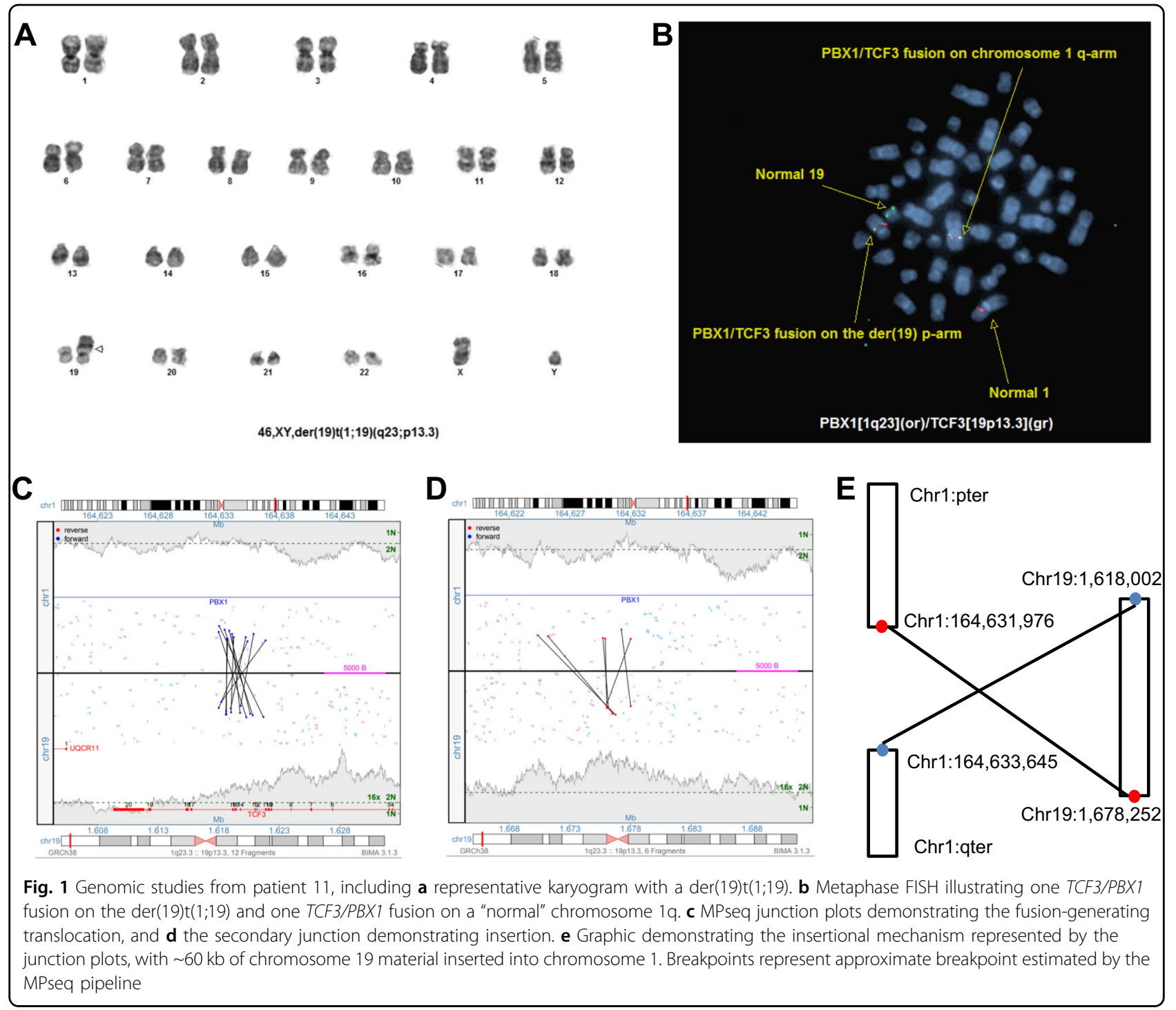

chromosome study in each case identified the more common unbalanced derivative chromosome 1;19 along with two apparently normal copies of chromosome 1 . Metaphase FISH studies using the TCF $3 / P B X 1$ probe set on the abnormal metaphases demonstrated the expected $T C F 3 / P B X 1$ fusion on the $\operatorname{der}(19) \mathrm{t}(1 ; 19)$, while the second $T C F 3 / P B X 1$ fusion was revealed on one of the apparently normal copies of chromosome 1 at the typical 1q23 location of $P B X 1$, suggesting a potential cryptic insertional rearrangement. MPseq clarified that an $\sim 30-60 \mathrm{~kb}$ segment of chromosome 19p was inserted into chromosome 1q, supporting the D-FISH results and resulting in a complex insertion involving two chromosomal regions containing the TCF3/PBX1 fusion. This insertional translocation and the derivative chromosome 19 show only a single breakpoint resulting in TCF3/PBX1 fusion, likely indicating an initial translocation event followed by a secondary "repair" event to restore the karyotypically normal chromosome 1. Alternatively, and less likely, insertional and translocation events with nearly identical breakpoints occurred independently, each resulting in a $T C F 3 / P B X 1$ fusion.

MPseq also revealed two cryptic TCF3/ZNF384 fusions resulting from unbalanced chromosome $19 p$ translocation events (patients 18 and 19). These cases resulted in $\sim 6 \mathrm{Mb}$ of chromosome 12 material replacing $\sim 2 \mathrm{Mb}$ of chromosome 19 material, a subtle change that is not readily detectable by conventional chromosome analysis. Additionally, since this rearrangement results in the loss of only a portion of the TCF3 probe signal using our $t(1 ; 19)$ D-FISH strategy, the only visible alteration was a diminished TCF3 signal.

In cases with additional TCF3 signals identified by interphase TCF3/PBX1 D-FISH studies, metaphase FISH 

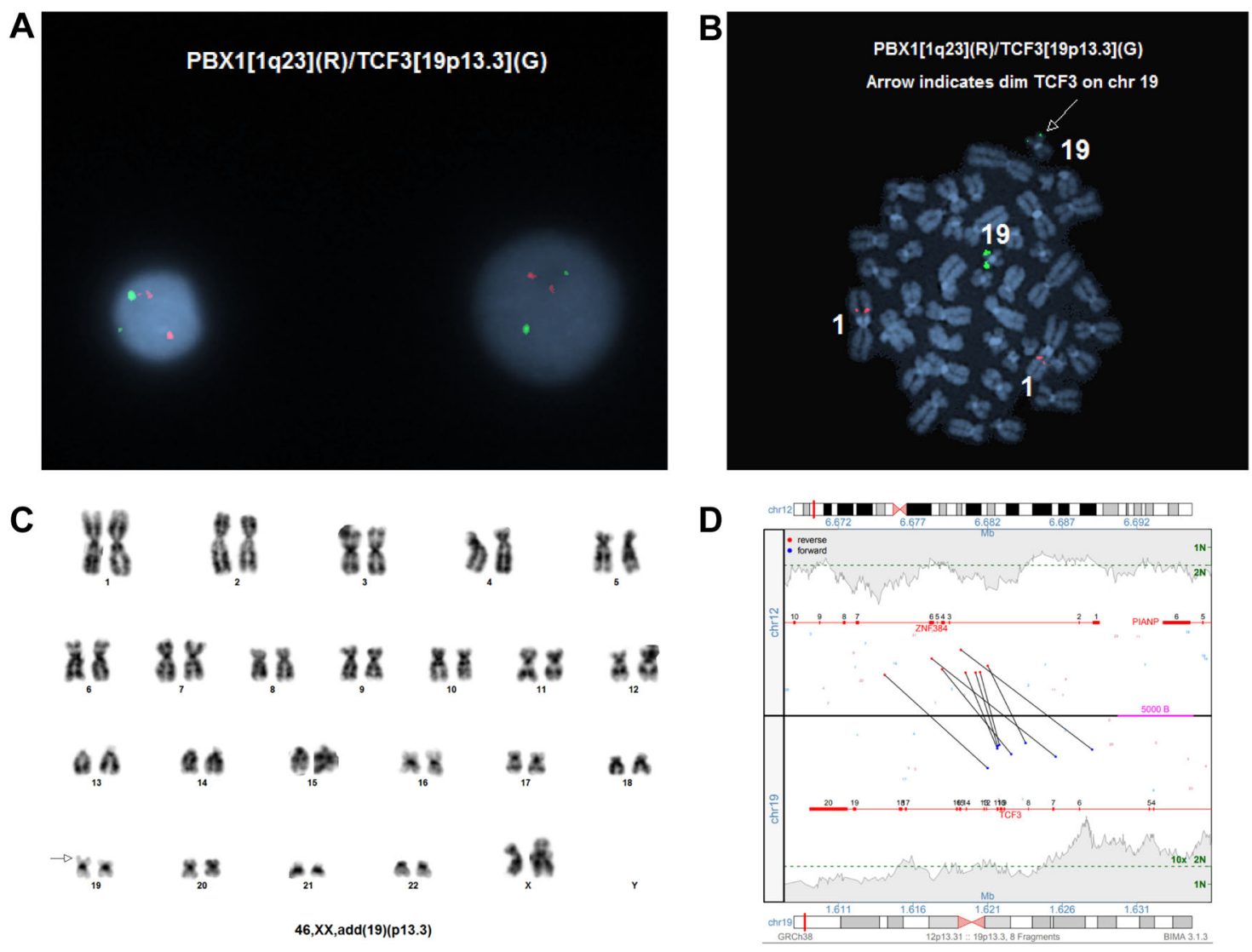

Fig. 2 Genomic studies from patient 18, including a representative interphase cells showing a diminished TCF3 signal suggesting a partial deletion or rearrangement of TCF3, using the TCF3/PBX1 D-FISH probe set. $\mathbf{b}$ Metaphase FISH demonstrating the diminished TCF3 signal is retained on chromosome 19p. c Representative karyogram with chromatin of undetermined origin attached to 19p. d MPseq junction plot demonstrating a TCF3/ZNF384 fusion

may provide low-resolution input into the general chromosomal locus of the TCF3 gene partner. For patients 14, 20 and 21, metaphase FISH localized the additional TCF3 FISH signal to chromosomes $12 \mathrm{p}, 22 \mathrm{q}$ and $11 \mathrm{q}$, respectively. However, for patients 15,16 and 17, metaphase FISH analysis was not possible due to either absence of metaphases or poor sample quality. Irrespective of metaphase FISH studies, which can at best suggest potential partner genes, MPseq was necessary to characterize each of the TCF3 gene fusion partners in nine cases evaluated in our series, including ZNF384 (patients 14-19), TEF (patient 20), FLI1 (patient 21) and HLF (patient 22).

MPseq therefore represents an advance in the detection of clinically relevant structural rearrangements ${ }^{21-23}$. While traditional NGS has the potential to detect rearrangements using paired end sequencing, MPseq allows for the characterization of structural rearrangements while requiring a significantly lower depth of coverage. The strength of MPseq lies in the unique library preparation, where long reads $(2-5 \mathrm{~kb})$ are circularized and fragmented to allow for their interrogation using traditional NGS. In contrast to basic NGS, where paired end sequencing will give linked reads separated by a few hundred base pairs, the circular MPseq fragments give linked reads much further away from each other $(2-5 \mathrm{~kb})$, thus significantly increasing the potential to identify discordant reads ${ }^{21}$.

The ability to assess genome-wide structure down to gene-level resolution makes MPseq a powerful tool for annotating simple and complex structural rearrangements as well as copy number changes, although some limitations exist. Breakpoints occurring within repetitive regions, such as in centromeric or segmental duplication mediated regions are challenging to map. The ability to use overall coverage as a proxy for copy number alleviates this limitation to some extent assuming any repeatmediated rearrangement is unbalanced. Similarly, its ability to detect terminal rearrangements is also limited since telomeric sequences are also repetitive and difficult to map, and are therefore subject to the same technical challenges. Lastly, the low depth of coverage of MPseq 

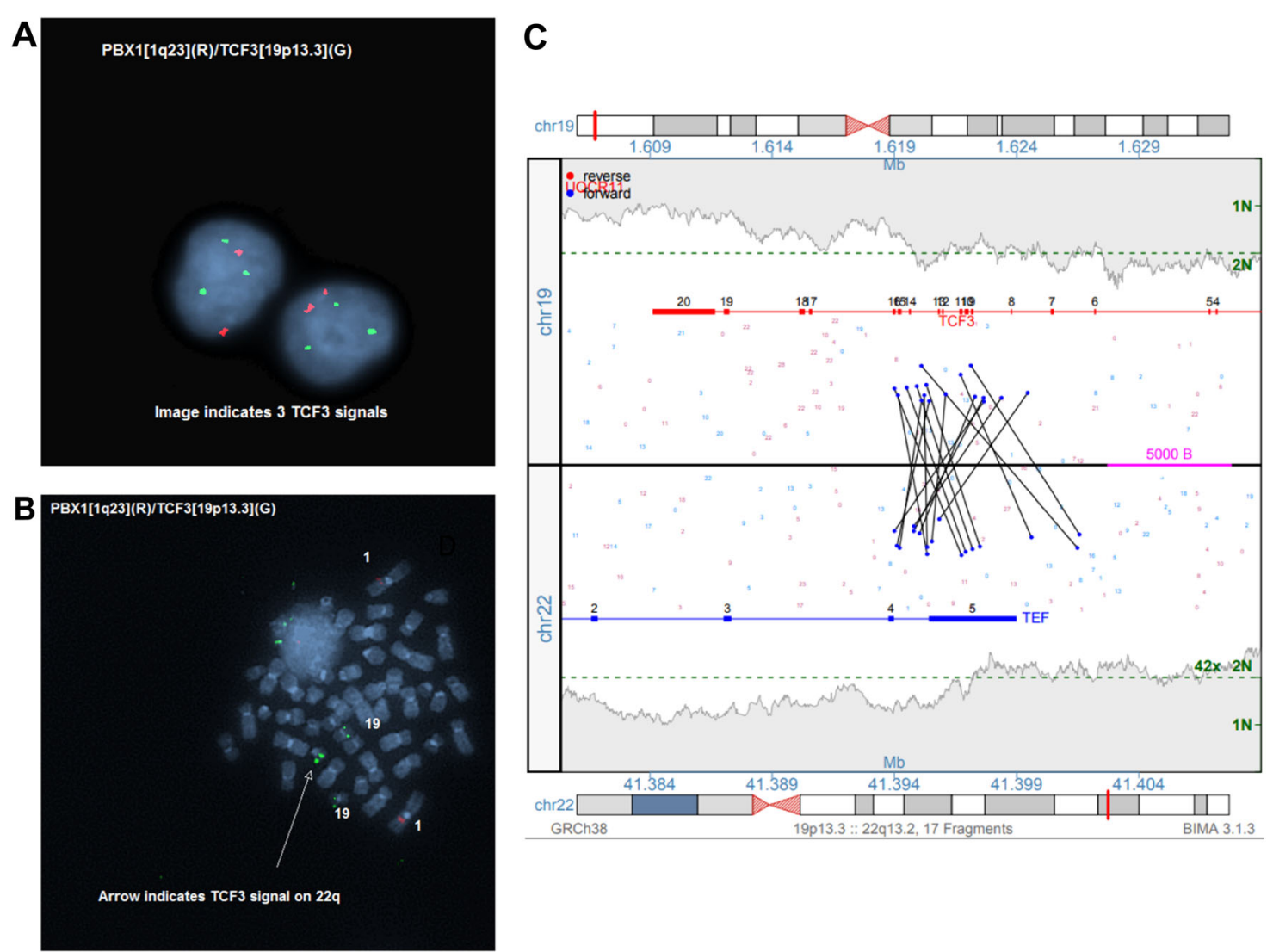

Fig. 3 Genomic studies from patient 20, including a interphase cells showing an extra TCF3 signal using the TCF3/PBX1 D-FISH probe set. b Metaphase FISH showing hybridization of TCF3 signals to both chromosomes 19, in addition to 22q. c MPseq junction plot demonstrating TCF3/TEF fusion

makes the detection of low level or subclonal rearrangements more challenging, with clonal populations at less than 25 and $10 \%$ involvement having reduced sensitivity for copy number and structural rearrangements, respectively $^{23}$. Therefore, MPseq offers the most utility in a diagnostic setting as opposed to monitoring for minimal residual disease. However, as the cost of sequencing continues to decline and instrument output increases, it is likely these limitations could be overcome with increased sequencing depth.

In conclusion, we have presented a 22-patient pediatric B-ALL/LBL cohort with TCF3 rearrangements that have been interrogated by standard cytogenetics, D-FISH for $T C F 3 / P B X 1$ and MPseq. Our data demonstrate the ability of MPseq to characterize cryptic structural rearrangements associated with discordant chromosome and TCF3/ PBX1 FISH results and to identify rare and novel TCF3 gene fusion partners associated with diminished or additional TCF3 FISH signals. We report the identification of a novel TCF3 fusion partner, TEF, by MPseq, and based on the functional similarities to $H L F, T E F$ may represent a second possible unfavorable TCF3 translocation. Overall,
MPseq represents a powerful NGS-based technology that allows for further investigation and characterization of structural chromosome rearrangements in hematologic malignancies and will aid in the overall understanding of genomic structural variation.

\section{Acknowledgements}

This study was supported by the Mayo Clinic Department of Laboratory Medicine and Pathology.

\section{Author details}

'Division of Laboratory Genetics and Genomics, Department of Laboratory Medicine and Pathology, Mayo Clinic, Rochester, MN, USA. ${ }^{2}$ Center for Individualized Medicine-Biomarker Discovery, Mayo Clinic, Mayo Clinic, Rochester, MN, USA. ${ }^{3}$ Department of Pathology, Johns Hopkins University School of Medicine, Baltimore, MD, USA

\section{Authors' contributions}

R.A.R., J.F.P., and R.P.K. wrote the manuscript; R.A.R., S.A.S., C.M.W., and Y.N. analyzed the raw data; S.A.S., C.M.W., G.V., J.B.S., Y.N., P.T.G., N.L.H., and L.B.B. reviewed and approved the final manuscript.

\section{Conflict of interest}

GV: Algorithms described in this manuscript for mate-pair sequencing are licensed to WholeGenome LLC owned by GV. 


\section{Publisher's note}

Springer Nature remains neutral with regard to jurisdictional claims in

published maps and institutional affiliations.

Received: 27 June 2019 Revised: 13 August 2019 Accepted: 15 August 2019 Published online: 01 October 2019

\section{References}

1. Mullighan, C. G. Molecular genetics of B-precursor acute lymphoblastic leukemia. J. Clin. Invest. 122, 3407-3415 (2012).

2. Andersen, M. K. et al. Paediatric B-cell precursor acute lymphoblastic leukaemia with $\mathrm{t}(1 ; 19)(q 23 ; \mathrm{p} 13)$ : clinical and cytogenetic characteristics of 47 cases from the Nordic countries treated according to NOPHO protocols. Br. J. Haematol. 155, 235-243 (2011).

3. Kager, L. et al. Incidence and outcome of TCF3-PBX1-positive acute lymphoblastic leukemia in Austrian children. Haematologica 92, 1561-1564 (2007).

4. Swerdlow, S. H. et al. WHO Classification of Tumours of Haematopoietic and Lymphoid Tissues Revised 4th edn. (International Agency for Research on Cancer, Lyon, France, 2017).

5. Paulsson, K., Horvat, A., Fioretos, T., Mitelman, F. \& Johansson, B. Formation of $\operatorname{der}(19) t(1 ; 19)(q 23 ; p 13)$ in acute lymphoblastic leukemia. Genes Chromosomes Cancer 42, 144-148 (2005).

6. Paulsson, K. et al. Characterization of genomic translocation breakpoints and identification of an alternative TCF3/PBX1 fusion transcript in $t(1 ; 19)$ (q23; p13)-positive acute lymphoblastic leukaemias. Br. J. Haematol. 138, 196-201 (2007).

7. Zhong, C. H. et al. E2A-ZNF384 and NOL1-E2A fusion created by a cryptic $t$ (12;19)(p13.3; p13.3) in acute leukemia. Leukemia 22, 723-729 (2008).

8. Barber, K. E. et al. Molecular cytogenetic characterization of TCF3 (E2A)/19p13.3 rearrangements in B-cell precursor acute lymphoblastic leukemia. Genes Chromosomes Cancer 46, 478-486 (2007).

9. Hirabayashi, S. et al. ZNF384-related fusion genes define a subgroup of childhood B-cell precursor acute lymphoblastic leukemia with a characteristic immunotype. Haematologica 102, 118-129 (2017).

10. Shearer, B. M., Flynn, H. C., Knudson, R. A. \& Ketterling, R. P. Interphase FISH to detect PBX1/E2A fusion resulting from the $\operatorname{der}(19) \mathrm{t}(1 ; 19)(\mathrm{q} 23 ; \mathrm{p} 13.3)$ or $\mathrm{t}(1 ; 19)$ (q23;p13.3) in paediatric patients with acute lymphoblastic leukaemia. Br. J. Haematol. 129, 45-52 (2005).

11. Dewald, G. W., Broderick, D. J., Tom, W. W., Hagstrom, J. E. \& Pierre, R. V. The efficacy of direct, 24-hour culture, and mitotic synchronization methods for cytogenetic analysis of bone marrow in neoplastic hematologic disorders. Cancer Genet. Cytogenet. 18, 1-10 (1985).

12. Drucker, T. M. et al. BIMA V3: an aligner customized for mate pair library sequencing. Bioinformatics 30, 1627-1629 (2014).

13. Johnson, S. H. et al. SVAtools for junction detection of genome-wide chromosomal rearrangements by mate-pair sequencing (MPseq). Cancer Genet. 221, 1-18 (2018).

14. Smadbeck, J. B. et al. Copy number variant analysis using genome-wide matepair sequencing. Genes Chromosomes Cancer 57, 459-470 (2018).

15. Kato, M. \& Manabe, A. Treatment and biology of pediatric acute lymphoblastic leukemia. Pediatr. Int. 60, 4-12 (2018).

16. Gu, Z. et al. Genomic analyses identify recurrent MEF2D fusions in acute lymphoblastic leukaemia. Nat. Commun. 7, 13331 (2016).

17. Aspland, S. E., Bendall, H. H. \& Murre, C. The role of E2A-PBX1 in leukemogenesis. Oncogene 20, 5708-5717 (2001).

18. Seidel, M. G. \& Look, A. T. E2A-HLF usurps control of evolutionarily conserved survival pathways. Oncogene 20, 5718-5725 (2001)

19. Hunger, S. P., Li, S., Fall, M. Z., Naumovski, L. \& Cleary, M. L. The proto-oncogene HLF and the related basic leucine zipper protein TEF display highly similar DNA-binding and transcriptional regulatory properties. Blood 87, 4607-4617 (1996).

20. Inukai, T. et al. TEF, an antiapoptotic bZIP transcription factor related to the oncogenic E2A-HLF chimera, inhibits cell growth by down-regulating expression of the common beta chain of cytokine receptors. Blood 105, 4437-4444 (2005)

21. Peterson, J. F. et al. Use of mate-pair sequencing (MPseq) to characterize a complex cryptic BCR/ABL1 rearrangement observed in a newly diagnosed case of chronic myeloid leukemia. Hum. Pathol. 89, 109-114 (2019).

22. Tran, A. N. et al. High-resolution detection of chromosomal rearrangements in leukemias through mate pair whole genome sequencing. PLOS ONE 13, e0193928 (2018).

23. Aypar, $U$. et al. Mate pair sequencing improves detection of genomic abnormalities in acute myeloid leukemia. Eur. J. Haematol. 102, 87-96 (2019). 